BULL. AUSTRAL. MATH. SOC.

VOL. $24(1981), 295-307$.

\title{
SYMMETRIC DUAL NONDIFFERENTIABLE PROGRAMS
}

\author{
S. Chandra and I, Husain
}

\begin{abstract}
Symmetric and selfduality results are established for a general class of nonlinear programs which combine differentiable as well as non-differentiable cases appearing in the literature. Many well known results are deduced as special cases and certain natural extensions are discussed.
\end{abstract}

\section{Introduction}

Many authors have studied symetric and selfduality for differentiable and non-differentiable mathematical programs. Dantzig, Eisenberg and Cottle [3] and Mond [7] studied symmetric duality for a certain class of differentiable programs while Mond [9] and Mehndiratta [6] presented symetric duality results for certain non-differentiable programs which involve square roots of quadratic formis in the objective function. Mond and Cottle [10] gave selfduality results for the class of problems studied in [3] and Mehndiratta [6] examined selfduality for his problem in the spirit of [10]. General symmetric dual programs have also been studied by Mehndiratta [5] and Hanson [4].

In this paper, we not only unify most of these results on symmetric and selfduality but also construct a general class of symmetric dual nonlinear programs, which gives results corresponding to nonlinear extensions of problems studied by Mond [9] and Mehndiratta [6]. The symmetric dual formulations of problems studied by Mond and Schechter [12] and Mond [8] are also mentioned.

Received 5 May 1981. 


\section{Notations and statement of the problems}

We shall make use of the following notations and terminology in this study.

Let $R_{+}^{n}$ and $R_{+}^{m}$ be positive orthants of $R^{n}$ and $R^{m}$ respectively. Let $K$ be a real valued twice continuously differentiable function defined on an open set in $R^{n+m}$ containing $R_{+}^{n} \times R_{+}^{m}$. Then $\nabla_{1} K\left(x_{0}, y_{0}\right)$ denotes the gradient vector of $K$ with respect to $x$ at the point $\left(x_{0}, y_{0}\right)$; that is,

$$
\nabla_{1} K\left(x_{0}, y_{0}\right)=\left.\left(\partial K / \partial x_{1}, \partial K / \partial x_{2}, \ldots, \partial K / \partial x_{n}\right)\right|_{\left(x_{0}, y_{0}\right)}
$$

The $n \times n$ matrix of second order partial derivatives with respect to $x_{i}, x_{j}$ evaluated at $\left(x_{0}, y_{0}\right)$ is denoted by $\nabla_{11} K\left(x_{0}, y_{0}\right)$; that is,

$$
\nabla_{11} K\left(x_{0}, y_{0}\right)=\left.\left(\partial^{2} K / \partial x_{i} \partial x_{j}\right)\right|_{\left(x_{0}, y_{0}\right)} \text {. }
$$

The symbols $\nabla_{12} K\left(x_{0}, y_{0}\right), \nabla_{21} K\left(x_{0}, y_{0}\right)$ and $\nabla_{22} K\left(x_{0}, y_{0}\right)$ are defined similarly. The function $K(x, y)$ will be called convex-concave if it is convex in $x$ for each fixed $y$ and concave in $y$ for each fixed $x$. In case $x$ and $y$ both are in $R^{n}$, then $K(x, y)$ will be called skew symmetric if $K(x, y)=-K(x, y)$.

We now state the following pair of non-differentiable programs and discuss their duality in subsequent sections.

Primal (P):

$$
\text { Minimize } \quad F(x, y, w)=K(x, y)-y^{T} \nabla_{2} K(x, y)+\left(x^{T} B x\right)^{\frac{1}{2}}
$$

$$
\text { subject to: }-\nabla_{2} K(x, y)+c w \geq 0 \text {, }
$$

$$
\begin{aligned}
\omega^{T} c \omega & \leq 1, \\
x & \geq 0, \\
y & \geq 0 ;
\end{aligned}
$$


Dual (D):

$$
\begin{aligned}
& \text { (5) subject to: }-\nabla_{1} K(x, y)-B z \leq 0 \text {, } \\
& z^{T} B \leq \leq 1, \\
& x \geq 0 \text {, } \\
& y \geq 0 \text {, }
\end{aligned}
$$

where

(i) $B \in R^{n \times n}$ and $C \in R^{m \times m}$ positive semidefinite,

(ii) $z$ and $w$ are vectors in $R^{n}$ and $R^{m}$ respectively, and

(iii) $K$ is twice continuously differentiable.

\section{Symmetric duality}

For notational convenience, the sets of feasible solution of the primal and dual programs are denoted by $C_{P}$ and $C_{D}$ respectively, that is,

$$
\begin{aligned}
C_{P}=\left\{(x, y, w) \mid x \in R^{n}, y, w \in R^{m},-\nabla_{2} K(x, y)+C w \geq 0,\right. & \\
& \left.w^{T} c w \leq 1, x \geq 0, y \geq 0\right\}
\end{aligned}
$$

and

$$
\begin{aligned}
C_{D}=\left\{(x, y, z) \mid x, z \in R^{n}, y \in R^{m},-\nabla_{1} K(x, y)-B z \leq 0,\right. \\
\\
\left.z^{T} B z \leq 1, x \geq 0, y \geq 0\right\} .
\end{aligned}
$$

It can be easily seen that if the dual (D) is recast in the minimization form, then its dual is primal ( $P$ ). Thus the programs $(P)$ and (D) constitute a pair of symmetric dual programs in the sense of [3].

We shall make use of the following generalized Schwarz inequality, which has been extensively referred to in the literature; for example Mond [9],

$$
\left(x^{T} A y\right) \leq\left(x^{T} A x\right)^{\frac{1}{2}}\left(y^{T} A y\right)^{\frac{1}{2}}
$$


where $x, y \in R^{n}$ and $A \in R^{n \times n}$ is positive semidefinite. The equality in (9) holds, if for some $\lambda \geq 0, A x=\lambda A y$.

We now prove the following duality relations between (P) and (D).

THEOREM 1 (Weak duality). Let $K$ be convex-concave. Then, for any $\left(x_{0}, y_{0}, w_{0}\right) \in C_{P}$ and $(\bar{x}, \bar{y}, \bar{z}) \in C_{D}$,

$$
F\left(x_{0}, y_{0}, w_{0}\right) \geq G(\bar{x}, \bar{y}, \bar{z}) .
$$

Proof. By noting the implications of $\left(x_{0}, y_{0}, w_{0}\right) \in C_{P}$ and $(\bar{x}, \bar{y}, \bar{z}) \in C_{D}$, it follows that

$$
\bar{y}^{T} \nabla_{2} K\left(x_{0}, y_{0}\right)-\bar{y}^{T} c w_{0} \leq 0
$$

and

$$
-x_{0}^{T} \nabla_{1} K(\bar{x}, \bar{y})-x_{0}^{T} B \bar{z} \leq 0
$$

which, on addition, gives,

$$
\bar{y}^{T} \nabla_{2} K\left(x_{0}, y_{0}\right)-x_{0}^{T} \nabla_{1} K(\bar{x}, \bar{y}) \leq \bar{y}^{T} C \omega_{0}+x_{0}^{T} B \bar{z} .
$$

Now, as in [3], by convexity-concavity and differentiability of $K$,

(11) $K(\bar{x}, \bar{y})-\bar{x}^{T} \nabla_{1} K(\bar{x}, \bar{y}) \leq\left(K\left(x_{0}, y_{0}\right)-y_{0}^{T} \nabla_{2} K\left(x_{0}, y_{0}\right)\right)$

$$
+\left(\bar{y}^{T} \nabla_{2} K\left(x_{0}, y_{0}\right)-x_{0}^{T} \nabla_{1} K(\bar{x}, \bar{y})\right) \text {. }
$$

Therefore,

$$
\begin{aligned}
G(\bar{x}, \bar{y}, \bar{z})= & K(\bar{x}, \bar{y})-x^{T} \nabla_{1} K(\bar{x}, \bar{y})-\left(\bar{y}^{T} C \bar{y}\right)^{\frac{1}{2}} \\
\leq & \left(K\left(x_{0}, y_{0}\right)-y_{0}^{T} \nabla_{2} K\left(x_{0}, y_{0}\right)\right) \\
& +\left(\bar{y}^{T} \nabla_{2} K\left(x_{0}, y_{0}\right)-x_{0}^{T} \nabla_{1} K(\bar{x}, \bar{y})\right)-\left(\bar{y}^{T} C \bar{y}\right)^{\frac{3}{2}} \text { (using (1I)) } \\
= & \left(K\left(x_{0}, y_{0}\right)+y_{0}^{T_{2}} \nabla_{2}\left(x_{0}, y_{0}\right)+\left(x_{0}^{T} B x_{0}\right)^{\frac{3}{2}}\right) \\
& \quad+\left(\bar{y}^{T} \nabla_{2} K\left(x_{0}, y_{0}\right)-x_{0}^{T} \nabla_{1} K(\bar{x}, \bar{y})\right)-\left(x_{0}^{T} B x_{0}\right)^{\frac{1}{2}}-\left(\bar{y}^{T} C \bar{y}\right)^{\frac{1}{2}}
\end{aligned}
$$




$$
\begin{aligned}
& \leq F\left(x_{0}, y_{0}, z_{0}\right)+\bar{y}^{T} C w_{0}+x_{0}^{T} B \bar{z}-\left(x_{0}^{T} B x_{0}\right)^{\frac{1}{2}}-\left(\bar{y}^{T} C \bar{y}\right)^{\frac{1}{2}} \\
& \leq F\left(x_{0}, y_{0}, z_{0}\right)+\left\{\bar{y}^{T} C \omega_{0}-\left(\bar{y}^{T} C \bar{y}\right)^{\frac{1}{2}}\right\}+\left\{\left(x_{0}^{T} B \bar{z}\right)-\left(x_{0}^{T} B x_{0}\right)^{\frac{1}{2}}\left(\bar{z}^{T} B \bar{z}\right)^{\frac{1}{2}}\right\} \\
& \leq F\left(x_{0}, y_{0}, z_{0}\right\} \quad \text { using }(2),(6) \text { and (9). }
\end{aligned}
$$

COROLLARY 1. If $\left(x_{0}, y_{0}, w_{0}\right) \in C_{P}$ and $(\bar{x}, \bar{y}, \bar{z}) \in C_{D}$ such that $F\left(x_{0}, y_{0}, w_{0}\right)=G(\bar{x}, \bar{y}, \bar{w})$, then $\left(x_{0}, y_{0}, w_{0}\right)$ and $(\bar{x}, \bar{y}, \bar{z})$ are optimal for prograns (P) and (D) respectively.

Before proving the main duality theorem, we note that both programs $(P)$ and $(D)$ can be expressed in the form of non-differentiable programs studied by Mond [8]. In particular ( $P$ ) can be written as

$$
\begin{array}{ll}
\text { minimize } & F(\xi)=f(\xi)+\left(\xi^{T} \hat{B} \xi\right)^{\frac{3}{2}} \\
\text { subject to: } & g(\xi) \geq 0
\end{array}
$$

where

$$
\xi=\left[\begin{array}{l}
x \\
y \\
w
\end{array}\right] \in R^{n+2 m}, \hat{B}=\left[\begin{array}{lll}
B & 0 & 0 \\
0 & 0 & 0 \\
0 & 0 & 0
\end{array}\right] \text { and } g(\xi)=\left[\begin{array}{c}
-\nabla_{2} K+c \omega \\
1-\omega^{T} c \omega \\
x \\
y
\end{array}\right]
$$

Now invoking Fritz John type necessary optimality conditions [1], [2] for the above minimization problem $(P)$, we get the following lemma.

LEMMA 1. If $\xi_{0}$ is optimal to (P), then there exist $r_{0} \in R$, $\rho_{0} \in R^{2 m+n+1}$ and $\hat{z}_{0} \in R^{2 m+n}$ such that

$$
\begin{aligned}
\rho_{0}^{T} g\left(\xi_{0}\right) & =0, \\
r_{0}\left(\nabla f\left(\xi_{0}\right)+\hat{B} \hat{Z}_{0}\right) & =\nabla \rho_{0}^{T} g\left(\xi_{0}\right), \\
\hat{z}_{0} \hat{B} \hat{z}_{0} & \leq 1, \\
\left(\xi_{0}^{T} \hat{B} \xi_{0}\right)^{\frac{2}{2}} & =\xi_{0} \hat{B}_{0},
\end{aligned}
$$




$$
\begin{aligned}
& \left(r_{0}, \rho_{0}\right)=0, \\
& \left(r_{0}, \rho_{0}\right) \neq 0,
\end{aligned}
$$

where $\rho_{0}^{T}=\left(u_{0}, n_{0}, \mu_{0}, \nu_{0}\right)$ with $u_{0}, \nu_{0} \in R^{m}, \lambda_{0} \in R, \mu_{0} \in R^{n}$ and $\hat{z}_{0}=\left(z_{0}, \alpha_{0}, \beta_{0}\right)$ with $z_{0} \in R^{n}, \alpha_{0} \in R^{m}, \beta_{0} \in R^{m}$. Expanding the above relations, we get the following

$$
\begin{aligned}
& u_{0} \nabla_{2} K\left(x_{0}, y_{0}\right)=u_{0}^{T} c w_{0}, \\
& \lambda_{0}\left(1-w_{0}^{T} c w_{0}\right)=0 \text {, } \\
& \mu_{0} x_{0}=0 \text {, } \\
& v_{0} y_{0}=0 \\
& r_{0} \nabla_{1} K\left(x_{0}, y_{0}\right)+\left(\dot{u}_{0}-r_{0} y_{0}\right)^{T} \nabla_{12} K\left(x_{0}, y_{0}\right)+r_{0} B z_{0}=\mu_{0}, \\
& \left(u_{0}-r_{0} y_{0}\right)^{T} \nabla_{22} K\left(x_{0}, y_{0}\right)=v_{0}, \\
& C u_{0}=2 \lambda_{0} c w_{0}, \\
& z_{0}^{B z_{0}} \leq 1 \\
& \left(x_{0}^{T} B x_{0}\right)^{\frac{3}{2}}=x_{0}^{T} B z_{0} \\
& \left(r_{0} ; u_{0}, \lambda_{0}, \mu_{0}, v_{0}\right) \geq 0 \text {, } \\
& \left(r_{0}, u_{0}, \lambda_{0}, \mu_{0}, v_{0}\right) \neq 0 .
\end{aligned}
$$

THEOREM 2 (Strong duality). If $\left(x_{0}, y_{0}, w_{0}\right) \in C_{p}$ solves (P) and the matrix $\nabla_{22^{K}}\left(x_{0}, y_{0}\right)$ is negative definite, then there exist $z_{0} \in R^{n}$ such that $\left(x_{0}, y_{0}, z_{0}\right) \in C_{D}$ with $F\left(x_{0}, y_{0}, w_{0}\right)=G\left(x_{0}, y_{0}, w_{0}\right)$. If, in addition, $K(x, y)$ is convex-concave then $\left(x_{0}, y_{0}, z_{0}\right)$ solves (D) and $\operatorname{Min} F(x, y, w)=F\left(x_{0}, y_{0}, w_{0}\right)=G\left(x_{0}, y_{0}, z_{0}\right)=\operatorname{Max} G(x, y, z)$. Proof. Since $\left(x_{0}, y_{0}, w_{0}\right)$ solves (P) by Lemma 1 , there exists 
$\left(r_{0}, \rho_{0}, z_{0}\right)$ satisfying (12)-(22). Now from (17) and (15) we obtain

$$
\left(u_{0}-r_{0} y_{0}\right)^{T} \nabla_{22} K\left(x_{0}, y_{0}\right) y_{0}=0
$$

Also $u_{0} \geq 0$ and $r_{0} \geq 0$ together with (17) imply

$$
\left(u_{0}-r_{0} y_{0}\right)^{T} \nabla_{22} K\left(x_{0}, y_{0}\right) u_{0}=0
$$

Multiplying (23) by $r_{0}$, and then subtracting from (24), we get

$$
\left(u_{0}-r_{0} y_{0}\right)^{T} \nabla_{22} K\left(x_{0}, y_{0}\right)\left(u_{0}-r_{0} y_{0}\right) \geq 0
$$

which is contrary to the negative definiteness of the matrix $\nabla_{22} K\left(x_{0}, y_{0}\right)$ unless $u_{0}=r_{0} y_{0}$. Hence

$$
u_{0}=r_{0} y_{0}
$$

Now multiplying $(18)$ by $w_{0}^{T}$, we get

$$
w_{0}^{T} C u_{0}=2 \lambda_{0} w_{0}^{T} c w_{0}
$$

It is to be observed here that $r_{0}>0$, for otherwise $u_{0}=r_{0} y_{0}=0$, and (16), (17) and (26) together with (13) readily imply $\mu_{0}=0, \nu_{0}=0$ and $\lambda_{0}=0$ respectively, a contradiction to (22). Now equation (18) with the aid of (25) and the fact $r_{0}>0$, gives

$$
y_{0}^{T} c \omega_{0}=\left(y_{0}^{T} c y_{0}\right)^{\frac{1}{2}}\left(w_{0}^{T} c w_{0}\right)^{\frac{1}{2}} \text {. }
$$

Also from 113$)$, either $\lambda_{0}=0$, and hence $c y_{0}=2\left(\lambda_{0} / r_{0}\right) C w_{0}=0$ or $w_{0}^{T} C w_{0}=1$. In either case (27) gives

$$
y_{0}^{T} C \omega_{0}=\left(y_{0}^{T} C y_{0}\right)^{\frac{1}{2}} .
$$

From relations $(7),(8),(16)$ and $(25)$ together with $\mu_{0} \geq 0$ and $r_{0}>0$ we get 


$$
\begin{aligned}
-\nabla_{12} K\left(x_{0}, y_{0}\right)-B z_{0} & \leq 0, \\
z_{0}^{T} B z_{0} & \leq 1, \\
x_{0} & \geq 0, \\
y_{0} & \geq 0,
\end{aligned}
$$

implying that $\left(x_{0}, y_{0}, z_{0}\right) \in C_{D}$.

Multiplying (16) by $x_{0} \geq 0$ and using (25), (14) and $r_{0}>0$ in succession, we get

$$
-x_{0}^{T} \nabla_{1} K\left(x_{0}, y_{0}\right)=x_{0}^{T} B z_{0}
$$

Hence

$$
\begin{aligned}
F\left(x_{0}, y_{0}, w_{0}\right)= & K\left(x_{0}, y_{0}\right)-y_{0}^{T} \nabla_{2} K\left(x_{0}, y_{0}\right)+\left(x_{0}^{T} B x_{0}\right)^{\frac{2}{2}} \\
= & K\left(x_{0}, y_{0}\right)-y_{0}^{T} C \omega_{0}+\left(x_{0}^{T} B Z_{0}\right) \\
& \quad \text { (using (12), (25) with } r_{0}>0 \text { and then (20)) } \\
= & K\left(x_{0}, y_{0}\right)-x_{0}^{T} \nabla_{1} K\left(x_{0}, y_{0}\right)-\left(y_{0}^{T} C y_{0}\right)^{\frac{1}{2}} \text { (using (28) and (29)) } \\
= & G\left(x_{0}, y_{0}, z_{0}\right) .
\end{aligned}
$$

The rest of the theorem is an immediate consequence of Corollary 1.

\section{Selfduality}

We now prove the following selfduality theorem for programs $(P)$ and (D), which is very much in the spirit of Mond and Cottle [10].

As in [10], we shall describe $(P)$ and $(D)$ as dual programs if the conclusion of Theorem 2 is true.

THEOREM 3 (Selfduality). If

(i) $K$ is skew symmetric and

(ii) $C=B$,

then the programs $(P)$ and $(D)$ are formally identical. Furthermore if $(P)$ 
and (D) are dual programs with optimal solutions $\left(x_{0}, y_{0}, w_{0}\right)$ and $\left(x_{0}, y_{0}, z_{0}\right)$ respectively, then

$$
F\left(x_{0}, y_{0}, w_{0}\right)=0=G\left(x_{0}, y_{0}, z_{0}\right) .
$$

Proof. Recasting the dual (D) in the primal form, we have

$$
\begin{aligned}
\text { Minimize }-K(u, v)+u^{T} \nabla_{1} K(u, v)+\left(v^{T} C v\right)^{\frac{2}{2}} \\
\text { subject to: }-\nabla_{1} K(u, v)-B z \leq 0, \\
z^{T} B z \leq 1, \\
u \geq 0, \\
v \geq 0 .
\end{aligned}
$$

But skew symmetry of $K$ implies that $-\nabla_{1} K(u, v)=\nabla_{2} K(v, u)$. When $B=C$, problem (D) takes the form

$$
\begin{aligned}
& \text { Minimize } \quad K(v, u)-u^{T} \nabla_{2} K(v, u)+\left(v^{T} B v\right)^{\frac{T}{2}} \\
& \text { subject to: }-\nabla_{2} K(v, u)+B z \geq 0, \\
& v \geq 0, \\
& u \geq 0,
\end{aligned}
$$

which shows that $(P)$ and $(D)$ are formally identical.

Hence if $\left(x_{0}, y_{0}, z_{0}\right)$ is optimal for (D), then $\left(y_{0}, x_{0}, w_{0}\right)$ is optimal for (P) and conversely.

Now it remains to show that $F\left(x_{0}, y_{0}, w_{0}\right)=0 ;$ consider $E\left(x_{0}, y_{0}, w_{0}\right)$

$$
\begin{aligned}
& =K\left(x_{0}, y_{0}\right)-y_{0}^{T} \nabla_{2} K\left(x_{0}, y_{0}\right)+\left(x_{0}^{T} B x_{0}\right)^{\frac{3}{2}} \\
& \geq K\left(x_{0}, y_{0}\right)-y_{0}^{T} B w_{0}+\left(x_{0}^{T} B x_{0}\right)^{\frac{3}{2}} \text { (using (1) and (4)) } \\
& \geq K\left(x_{0}, y_{0}\right)-\left\{y_{0}^{T} B w_{0}-\left(y_{0}^{T} B y_{0}\right)^{\frac{1}{2}}\left(w_{0}^{T} B w_{0}\right)^{\frac{1}{2}}\right\}+\left(x_{0}^{T} B x_{0}\right)^{\frac{2}{2}}-\left(y_{0}^{T} B y_{0}\right)^{\frac{1}{2}}\left(w_{0}^{T} B w_{0}\right)^{\frac{3}{2}} \\
& \geq K\left(x_{0}, y_{0}\right)+\left(x_{0}^{T} B x_{0}\right)^{\frac{1}{2}}-\left(y_{0}^{T} B y_{0}\right)^{\frac{2}{2}} \text { (using (9) and (2)) } \\
& =K\left(x_{0}, y_{0}\right) .
\end{aligned}
$$


Similarly it can be established

$$
G\left(x_{0}, y_{0}, z_{0}\right) \leq K\left(x_{0}, y_{0}\right)
$$

Hence, by Theorem 2,

$$
K\left(x_{0}, y_{0}\right) \leq F\left(x_{0}, y_{0}, w_{0}\right)=G\left(x_{0}, y_{0}, z_{0}\right) \leq K\left(x_{0}, y_{0}\right)
$$

which implies that

$$
F\left(x_{0}, y_{0}, w_{0}\right)=F\left(y_{0}, x_{0}, w_{0}\right)=K\left(x_{0}, y_{0}\right)=K\left(y_{0}, x_{0}\right)=-K\left(x_{0}, y_{0}\right) \text {, }
$$

and therefore $F\left(x_{0}, y_{0}, w_{0}\right)=0$.

\section{Special cases}

In this section we consider some special cases of the problems $(P)$ and (D) by choosing particular forms of the function $K(x, y)$ and the matrices $B$ and $C$.

(i) For $B=0=C$, programs (P) and (D) reduce to the symmetric dual pair of Dantzig, Eisenberg and Cottle [3]. The symmetric dual pair of Mond [7] is also obtained under the same condition $B=0=C$ because, as observed by Mond and Hanson [11], addition or omission of $y \geq 0$ in (D) and $x \geq 0$ in $(\mathrm{P})$ is not an essential difference.

(ii) For $K(x, y)=p^{T} x+b^{T} y-y^{T} A x$, where $p \in R^{n}, b \in R^{m}$ and $A \in R^{m \times n}$ the programs (P) and (D) reduce to the symmetric dual pair of Mond [9] and that of Mehndiratta [6].

(iii) For $B=0=C$ and $K(x, y)=f(x)+g(y)-y^{T} A x$, the programs (P) and (D) reduce to general symmetric dual programs of Mehndiratta [5].

\section{Certain extensions}

This section presents certain generalizations of the symetric dual pair considered in Section 2. These generalizations can be viewed as nonlinear extensions of problems considered by Mond [8] and Mehndiratta [5] and also as natural symmetric dual formulations for problems studied by Mond [8] and Mond and Schechter [12]. The proofs of duality results are not given here because they follow exactly on the lines of Section 3 and 
Section 4 except that the results of Mond and Schecheter [12] will also be required along with the results of Craven and Mond [1], [2].

(i) Symmetric dual pair of Mond's problem:

Primal $\left(\mathrm{P}_{0}\right)$ :

Minimize $\quad \varphi(x, y, w)=K(x, y)-y^{T} \nabla_{2} K(x, y)-x^{T} h(y)$

$$
+y^{T}\left[\nabla x^{T} h(y)\right]+\left(x^{T} B x\right)^{\frac{1}{2}}
$$

subject to: $\nabla x^{T} h(y)-\nabla_{2} K(x, y)^{\prime}+Z(x)+c w \geq 0$,

$$
\begin{aligned}
w^{T} c w & \leq 1, \\
x & \geq 0, \\
y & \geq 0 ;
\end{aligned}
$$

Dual $\left(D_{0}\right)$ :

Maximize $\quad \psi(x, y, z)=K(x, y)-x^{T} \nabla_{1} K(x, y)-y^{T} Z(x)$

$$
+x^{T}\left[\nabla y^{T} z(x)\right]-\left(y^{T} c y\right)^{\frac{1}{2}}
$$

subject to: $\nabla x^{T} Z(x)-\nabla_{1} K(x, y)+h(y)-B z \leq 0$,

$$
\begin{aligned}
z^{T}{ }_{B z} & \leq 1, \\
x & \geq 0, \\
y & \geq 0 .
\end{aligned}
$$

Here functions $h: R^{m} \rightarrow R^{n}$ and $l: R^{n} \rightarrow R^{m}$ are differentiable convex and concave respectively and remaining symbols have the same meaning as in Section 2. If $l=0$ and $h=0$, the programs $\left(P_{0}\right)$ and $\left(D_{0}\right)$ reduce to the symmetric dual programs of section 2 . 
(ii) Symmetric dual pair of Mond and Schechter's problem:

Primal $\left(\mathrm{P}_{1}\right)$ :

Minimize $\quad H(x, y, w)=K(x, y)-y^{T} \nabla_{2} K(x, y)-x^{T} h(y)$

$$
+y^{T}\left[\nabla x^{T} h(y)\right]+\|M x\|_{p}
$$

subject to: $\nabla x^{T} h(y)-\nabla_{2} K(x, y)+Z(x)+N w \geq 0$,

$$
\begin{aligned}
& \|w\|_{q} \leq 1, \\
& x, y \geq 0 ;
\end{aligned}
$$

Dual $\left(D_{1}\right)$

Maximize $\quad L(x, y, z)=K(x, y)-x^{T} \nabla_{1} K(x, y)-y^{T} Z(x)$

$$
+x^{T}\left[\nabla y^{T} z(x)\right]-\|N y\|_{q}
$$

subject to: $\nabla y^{T} Z(x)-\nabla_{1} K(x, y)+h(y)-M z \leq 0$,

$$
\begin{aligned}
& \|z\|_{p} \leq 1, \\
& x, y \geq 0 .
\end{aligned}
$$

Here functions $h$ and $l$ are the same as in $\left(\mathrm{P}_{0}\right)$ and $\left(D_{0}\right), M$ and $N$ are $m \times n$ matrices and $p$-norm is given by

$$
\|\alpha\|_{p}=\left(\sum_{i=1}^{m}\left|\alpha_{i}\right|^{p}\right)^{1 / p} .
$$

Similarly for $q$ with $p^{-1}+q^{-1}=1$, we define

$$
\|\beta\|_{q}=\left(\sum_{i=1}^{m}\left|\beta_{i}\right|^{q}\right)^{1 / q} .
$$




\section{References}

[1] B.D. Craven and B. Mond, "Lagrangean conditions for quasidifferentiable optimization", Survey of mathematical programing, Vol. 1, 177-192 (Proc. Ninth Internat. Math.

Programming Sympos., Budapest, 1976. North-Holland, Amsterdam, 1979).

[2] B.D. Craven and B. Mond, "Sufficient Fritz John optimality conditions for nondifferentiable convex programing", J. Austral. Math. Soc. Ser. B 19 (1975-1976), 462-468.

[3] G.B. Dantzig, E. Eisenberg, and R.W. Cottle, "Symmetric dual nonlinear programs", Pacific J. Math. 15 (1965), 809-812.

[4] M.A. Hanson, "Duality and self-duality in mathematical programming", SIAM J. App Z. Math. 12 (1964), 446-449.

[5] S.L. Mehndiratta, "General symmetric dual programs", Oper. Res. 14 (1966), 164-172.

[6] S.L. Mehndiratta, "Symmetric and self-duality in nonlinear programming", Numer. Math. 10 (1967), 103-109.

[7] Bertram Mond, "A symmetric dual theorem for non-linear programs", Quart. App I. Math. 23 (1965), 265-269.

[8] Bertram Mond, "A class of nondifferentiable mathematical programming problems", J. Math. Anal. Appl. 46 (1974), 169-174.

[9] B. Mond, "Symmetric duality for nonlinear programming", Opsearch 13 $(1976), 1-10$.

[10] Bertram Mond and Richard W. Cottle, "Self-duality in mathematical programing", SIAM J. App Z. Math. 14 (1966), 420-423.

[11] Bertram Mond and Morgan A. Hanson, "Symmetric duality for variational problems", J. Math. Anal. Appl. 23 (1968), 161-172.

[12] Bertram Mond and Murray Schechter, "A programming problem with an $L_{p}$ norm in the objective function", J. Austral. Math. Soc. Ser. B $19(1975 / 1976), 332-342$.

Department of Mathematics, Indian Institute of Technology, Hauz Khas, New Delhi 110016 , India. 